\title{
La « dimension argumentative » plurisémiotique du livre enrichi
}

The enhanced book and its plurisemiotic "argumentative dimension"

Sara Amadori

\section{(2) OpenEdition \\ 1 Journals}

\section{Édition électronique}

URL : http://journals.openedition.org/aad/2526

DOI : $10.4000 /$ aad. 2526

ISSN : 1565-8961

Éditeur

Université de Tel-Aviv

\section{Référence électronique}

Sara Amadori, «La « dimension argumentative » plurisémiotique du livre enrichi », Argumentation et Analyse du Discours [En ligne], 20 | 2018, mis en ligne le 15 avril 2018, consulté le 23 septembre 2019. URL : http://journals.openedition.org/aad/2526; DOI : 10.4000/aad.2526

Ce document a été généré automatiquement le 23 septembre 2019.

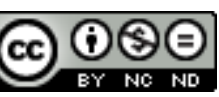

Argumentation \& analyse du discours est mis à disposition selon les termes de la licence Creative Commons Attribution - Pas d'Utilisation Commerciale - Pas de Modification 4.0 International. 


\title{
La « dimension argumentative » plurisémiotique du livre enrichi
}

The enhanced book and its plurisemiotic "argumentative dimension"

\author{
Sara Amadori
}

\section{Introduction}

1 La valeur heuristique de la littérature numérique, à savoir de toute œuvre littéraire créée pour n'être reçue que sur un support numérique en raison de sa dimension multimédia, animée ou interactive (Bouchardon 2014), offre une perspective de réflexion nouvelle et féconde non seulement pour les spécialistes en Sciences de l'Information et de la Communication, mais également pour tous ceux qui étudient l'argumentation rhétorique (Amossy 2010a). Dans cet article je focaliserai mon attention sur ce qu'on appelle désormais «livre enrichi », une évolution du " récit littéraire interactif" né sur le web (Bouchardon 2014, 2005)². La diffusion extraordinaire de nouveaux supports de lecture (tablettes, smartphones, etc.) a incité les éditeurs à développer des contenus culturels permettant d'exploiter les potentialités de ces nouveaux dispositifs. De grands éditeurs tels que Gallimard ou Seuil, ainsi que des pure players, ont commencé à introduire sur le marché français des produits numériques qui offrent une expérience de lecture originale, en raison de leur caractère multi-sensoriel et plurisémiotique (Saemmer 2015).

2 Le corpus de mon étude se compose de deux livres enrichis: Beauvoir, l'enquête, par Irène Frain (coédition Storylab-Lafon), et Alienare, par Chloé Delaume (paru au Seuil) ${ }^{3}$. Beauvoir, l'enquête de Frain est un roman bref qui se présente comme une enquête, enrichie d'extraits de films, musiques, notes de travail, photos inédites, images d'archives, ayant pour but d'accompagner le lecteur dans les coulisses de l'écriture de Beauvoir in love (Lafon). Delaume, auteure de littérature expérimentale et écrivaineblogueuse, publie quant à elle Alienare en collaboration avec Franck Dion et Sophie Couronne. Il s'agit d'un livre hybride qui mélange texte écrit, film et musique, à travers lequel l'écrivaine recherche une «forme d'art total» (Delaume 2015a : en ligne). 
Ces deux livres enrichis invitent à approfondir la réflexion concernant la distinction proposée par Amossy entre « dimension » et « visée argumentative ». Si cette dernière caractérise toute prise de parole qui défend explicitement une thèse et entend agir sur l'auditoire, la première est «l'orientation plus ou moins marquée de l'énoncé qui invite l'autre à partager des façons de penser, de voir, de sentir» (Amossy 2010a: 9). L'hétérogénéité sémiotique de ces nouvelles productions littéraires permet de mettre en évidence que la dimension non seulement verbale mais également visuelle, iconique-textuelle, audiovisuelle, sonore et musicale de ces œuvres possède un potentiel d'« argumentativité ».

4 Je me propose d'analyser les relations qui s'instaurent entre matérialité discursive, dispositif d'énonciation, "ethos éditorial» (Maingueneau 2013: 23) et «images d'auteures» (Amossy 2009 : en ligne) de Frain et de Delaume, afin de montrer combien ces dimensions contribuent à déterminer l'«argumentativité » de ces œuvres à un premier niveau para-péritextuel. En puisant dans la réflexion de la rhétorique du texte numérique (Saemmer 2015), je montrerai que la tentative d'orienter la réception de ces produits littéraires de la part aussi bien des éditeurs que des écrivaines est évidente. Une enquête (Tréhondart 2014, 2013) menée auprès des responsables des principales maisons d'édition qui produisent ce genre de livres a montré qu'ils ont intérêt à faire croire au public qu'il existe un véritable rapport de filiation entre ces produits et le livre en version papier. Une telle ressemblance est "rassurante » pour le lecteur, et permet de démentir les critiques suscitées par les pratiques de lecture fragmentaire traditionnellement associées au numérique (Saemmer 2011). En croisant la réflexion proposée par l'analyse du discours littéraire (Maingueneau 2004) avec l'approche développée par l'argumentation dans le discours (Amossy 2010a), je montrerai également que les éditeurs exploitent l'«image d'auteure » extratextuelle de Frain et de Delaume afin de confirmer la valeur littéraire de ces livres numériques. Dans l'« espace associé » à leur Opus (Maingueneau 2004: 113), les auteures elles-mêmes insistent aussi bien sur le caractère littéraire de ces œuvres que sur l'originalité et la valeur artistique ajoutée qui résulte de l'exploitation des ressources multimédia. Elles retravaillent ainsi partiellement leurs «ethè préalables» (Amossy 2010b), en se présentant au public comme des écrivaines innovantes et désireuses de renouveler les formes de leur création artistique dans une société qui change rapidement.

5 En faisant jouer l'«image d'auteure » extratextuelle de Frain et de Delaume avec l'« ethos auctorial» (Amossy 2009: en ligne) projeté dans leurs œuvres, je mettrai en évidence que des degrés de «subjectivité » ou d'« objectivité » différents (Amossy et Koren 2004) caractérisent les prises de parole des deux écrivaines, et qu'une telle gradualité influence le caractère plus ou moins implicite de leurs démarches argumentatives. L'étude de leurs modes d'énonciation à partir de la distinction proposée par Soulages entre dispositifs de médiatisation " de monstration ", " fictionnel " et " de mise en spectacle » (qui permettent respectivement un mode d'énonciation "authentifiant ", "fictif» et "ludique», 2007 : 21-23) jettera ainsi la lumière sur la façon dont la complexité plurisémiotique de leurs discours littéraires peut se mettre au service d'une « dimension argumentative » plus ou moins marquée. 


\section{1. «Argumentativité » de l'« image d'auteur » et de «l' ethos éditorial »}

\subsection{Une « image d'auteur » à la fois traditionnelle et innovante}

6 La première question à laquelle j'essaierai de répondre est de savoir pourquoi les éditeurs ont choisi deux auteures telles que Frain et Delaume. S'il ne s'agit pas de deux " auteures-auctors", selon la définition de Maingueneau (2009: en ligne), ces deux écrivaines sont néanmoins bien positionnées dans le champ littéraire en tant qu'« auteures-actrices » (Maingueneau 2009 : en ligne). Elles publient chez des maisons d'édition reconnues, ont reçu des prix littéraires, et leurs ouvrages ont fait l'objet de travaux universitaires. Un processus de constitution d'une "image d'auteure " (Amossy 2009 : en ligne) et de canonisation de leur Opus (Mainguenau 2004) est donc en cours.

7 Frain est agrégée de Lettres classiques, romancière et journaliste/portraitiste à Paris Match. Elle a publié une trentaine de livres, chez des éditeurs prestigieux tels que Seuil, Lafon, Fayard. Ses romans ont toujours connu un grand succès auprès du public. Selon Grégoire, elle « compte parmi les cinq écrivains les plus lus en France» (1993: 66). Les Naufragés de l'île Tromelin a reçu en 2009 le Grand Prix Palatine, un prix attribué aux ouvrages qui font preuve d'une grande rigueur historique, d'un sens aigu du romanesque et d'une évidente qualité littéraire. Ses livres sont souvent précédés de longues recherches, pour lesquelles Frain met en œuvre sa formation d'universitaire, de chercheuse et de journaliste.

8 Cette « image d'auteure » produite par le circuit éditorial et académique est rassurante pour un éditeur à la recherche de formats nouveaux. Il en est de même quant à l'engagement de Delaume. Avant tout romancière, Delaume a publié une vingtaine de romans, dans des maisons d'édition telles que Seuil, Léo Scheer ou Verticales. Elle a reçu le prix Décembre en 2001 pour Le Cri du sablier, son deuxième roman. Elle a été chroniqueuse à l'émission télévisée Arrêt sur image et crée des fictions radiophoniques pour France Culture. Elle est performeuse, pratique l'expérimentation instrumentale et travaille en solo ou en collaboration avec d'autres performeurs, chanteurs ou cinéastes. Bien que ses romans autofictifs appartiennent à la tradition littéraire papier, elle explore tous les médias contemporains pour faire circuler ses histoires et ses images d'elle-même (Ducas 2010 : 177).

Dans le cadre de son projet autofictif ${ }^{4}$ visant à dépasser le trauma familial (son père a tué sa mère et s'est ensuite suicidé en sa présence, elle avait alors dix ans), Delaume a choisi un pseudonyme littéraire qu'elle a substitué à son identité réelle (Nathalie-Anne Abdallah) et qui fait d'elle la fille adoptive de Boris Vian et d'Antonin Artaud. L'autofiction, telle que l'écrivaine la pratique, prend la forme d'un véritable laboratoire. Littérarité et expérimentation sont indissociables dans son «image d'auteure » :

Dans mon laboratoire, je cherche des outils pour produire de l'autofiction. Des outils autres que l'écriture, des outils plus technologiques. La musique électronique, internet, les jeux vidéo. [...] Chaque livre est un chantier [...] Musique et poésie sonore, chercher encore d'autres supports, fouiller dans la boîte à outils. Ratisser les zones en jachère, les territoires non exploités (Delaume 2008 : en ligne). 
Delaume, «depuis longtemps frustrée par le livre papier»(2015a: en ligne), non seulement est prête à s'ouvrir au numérique, mais elle est convaincue que la tablette rend possible la création d'un objet littéraire hybride, ayant une forme esthétique enfin accomplie (Delaume 2009). Il est aisé de comprendre pourquoi Flore Roumens, qui dirige la collection "Numérique » au Seuil, a proposé à Delaume de réaliser un livre enrichi. L'écrivaine a accepté avec enthousiasme : cela lui permettait de confirmer son « ethos préalable» (Amossy 2010b). C'est ainsi qu'Alienare a vu le jour.

11 Frain, quant à elle, confirme et retravaille son «ethos préalable" par le recours au numérique, qui permet de partager avec son lecteur son expérience d'écriture de façon nouvelle, plus intense. En effet, le support technique lui offre la possibilité de rendre son ethos de chercheuse et de journaliste encore plus convaincant. «Je suis formée par la Sorbonne [...] Je suis agrégée en lettres classiques, donc les bibliothèques, les archives, je sais comment on fait ", a-t-elle affirmé lors d'un entretien radiophonique (avec Nathalie de Broc). De même, elle insiste sur sa formation journalistique: «l'enquête du journaliste sur le terrain, que j'avais commencé à apprendre avec mes copains de Paris Match, je m'étais aperçue que c'était quelque chose de très rigoureux et aussi très physique " (avec Hubert Prolongeau). Cette "image d'auteure » que Frain projette d'elle-même trouve, dans la réalisation de Beauvoir, l'enquête son accomplissement, comme l'écrivaine le confirme :

Pour écrire mon livre, j'avais enquêté entre Paris, Chicago et les archives d'une université de l'ohio. J'avais vite accumulé un trésor de pépites documentaires inconnues, filmé, photographié, enregistré, noirci sur le vif des pages d'esquisses et de notes. Et vécu un véritable petit polar littéraire, car la vraie histoire des amants ne coïncidait jamais avec la version officielle, celle qui faisait de Sartre le seul vrai grand amour de Beauvoir. [...] Mes lecteurs [...] n'ont pas cessé de me demander de leur relater cette palpitante enquête. Seul le numérique permettait ce partage [...]. [Et] après cette aventure, je me sens infiniment mieux inscrite dans le sillon multimillénaire de la littérature (2014a : en ligne).

12 Le mouvement oscillatoire, entre tradition et innovation, de ce travail de "réglage » (Maingueneau 2004: 113) inscrit les créations numériques de Frain et de Delaume de façon singulière et captivante dans le panorama des productions littéraires. Les éditeurs Lafon/Storylab et Seuil, en choisissant deux écrivaines qui jouissent de ce que Maingueneau définirait comme un fort "coefficient d'“auctorité" " (2009: en ligne), ont voulu orienter efficacement la réception de ces produits. La littérarité que le lecteur est disposé à attacher préalablement à ces deux livres enrichis est, en effet, la conséquence du charisme et du prestige des deux auteures dans le champ littéraire. Autrement dit, leurs «images d'auteures» extratextuelles ont un potentiel argumentatif que l'on pourrait définir comme intrinsèque et qui joue un rôle important dans la construction de la "dimension argumentative» para-péritextuelle de ces nouvelles productions littéraires (comme on va le voir).

\section{2. «Argumentativité » de l'« ethos éditorial »}

$13 \mathrm{Au}$ croisement entre les théories de la réception venant des sciences du texte, les S.I.C. et l'approche de l'analyse du discours et de l'argumentation, je me pencherai maintenant sur la « dimension argumentative » de ce que Maingueneau appelle « l'ethos éditorial », «qui ressort de la collection où est publié le livre, du papier utilisé, de la couverture » (2013: 23). Cette notion est proche de celle d'« énonciation éditoriale», 
élaborée par Souchier et Jeanneret (2005)5. Dans ce cadre, la rhétorique de la réception développée par Saemmer (2015) s'est révélée essentielle. Sa démarche s'inscrit dans la filiation de la Nouvelle rhétorique de Perelman et Olbrechts-Tyteca (2000[1958]) et des théories de la réception d'Iser (1995) et de Jauss (2010). Or, selon Jauss, la réception esthétique d'une œuvre présuppose un contexte d'expériences antérieures (par exemple la connaissance des genres, des formes et des thématiques des œuvres précédentes), et s'inscrit toujours dans un tel contexte (2010:54-56). La « rhétorique de la réception " se propose ainsi de "cerner les pratiques de lecture modélisées par le texte numérique, à travers ses procédés rhétoriques et ses formes graphiques sur la page-écran » (Saemmer 2015 : 13). En concevant la lecture du texte numérique comme le résultat de la rencontre entre des pratiques de lecture prédéterminées par le texte lui-même et des imaginaires individuels et collectifs qui forment l'horizon d'attente de chaque lecteur, Saemmer $(2015: 63)$ incite à penser le destinataire d'un livre enrichi en termes d'« auditoire universel» (Perelman \& Olbrechets-Tyteca 2000). En effet, cette spécialiste considère le destinataire d'une œuvre de littérature numérique comme un « lecteur modèle " (selon la définition de Eco, Saemmer 2011), dont la réception sera prédéterminée par le texte lui-même et par les formes spécifiques de sa matérialité sémiotique. Le choix d'un format éditorial acquiert par conséquent une «dimension argumentative » dans la mesure où celui-ci apparaît comme "une conséquence du dialogisme inhérent au discours » (Amossy 2010a : 33).

En effet, le recours de la part des éditeurs à certaines "formes modèles", des «protocoles de lecture qui contribuent au potentiel d'action d'un texte » et qui font appel à des formats éditoriaux plus anciens (Saemmer 2015 : 119), vise à éveiller chez le lecteur l'imaginaire du livre imprimé et à confirmer l'appartenance des œuvres numériques à la tradition littéraire. La couleur, la forme, la taille des caractères, la pagination, la couverture, le sommaire, ainsi que toutes sortes d'allusions éditoriales entretenant un rapport 'dialogique' aux supports de lecture précédents, orientent l'acte de réception. Le format éditorial peut également surprendre et rompre intentionnellement avec l'imaginaire de la lecture sur support papier. Il est nécessaire alors d'évaluer quelles sont les conséquences de tels choix éditoriaux.

Mon corpus offre la possibilité de montrer que «l'ethos éditorial » fait écho aux images d'auteure respectives de Frain et de Delaume. Dans le cas de Frain, la volonté de s'inscrire dans l'héritage du livre papier est manifeste: son texte préfigure un public habitué à la lecture de ses romans. Beauvoir, l'enquête est lu sur l'application iBooks de l'iPad, son paratexte fait explicitement allusion à celui d'un livre papier. Sa couverture présente le nom de l'auteure et le titre de l'ouvrage, ainsi qu'une bande rouge indiquant au lecteur qu'il a reçu la «mention spéciale du jury du prix du livre numérique $2014 \%$. La tentative d'évoquer l'objet physique par ces marques d'énonciation éditoriale est incontestable, d'autant plus que la page-écran de la couverture, d'une couleur sépia, imite le papier usé par le temps.

Dans le frontispice, le lecteur trouve le nom des éditeurs, l'ISBN, la date du dépôt légal, et il découvre que l'ouvrage a été réalisé avec le soutien du Centre National du Livre. La forme-modèle du sommaire est remédiatisée de deux façons différentes, sous forme de liste sémantique des chapitres et de «chemin de fer » illustré en guise de vignettes de pages. Le lecteur a ainsi toujours la sensation de pouvoir se repérer facilement pendant la lecture de l'ouvrage, et de pouvoir en faire une expérience immersive et réfléchie. 

livre aux lecteurs), qui n'est que faiblement innovante par rapport aux conventions du genre. Le livre est divisé en chapitres, les pages sont numérotées. Tout ce qui pourrait éveiller l'expérience de la lecture sur papier est là. L'éditeur, en multipliant les efforts pour imiter la matérialité du codex, arrive même à altérer l'objet numérique en lui donnant un aspect artisanal. L'image 1 (Frain 2014b : 25) montre par exemple la tache qu'aurait laissée une tasse de café pendant les longues et épuisantes recherches menées par l'auteure pour écrire son Beauvoir in love.

La dimension typographique est également intéressante. À côté de caractères plus traditionnels qui éveillent l'imaginaire de l'écriture imprimée, d'autres imitent une écriture manuscrite. Un exemple de ce type de caractère est visible toujours dans l'image 1 (2014b : 25). Les pages $24-29$ sont présentées comme un extrait du « carnet de notes » de Frain (23) par une notation précédente de l'auteure, qui est écrite en marge de la page 23 sur un post-it jaune, avec le même caractère typographique que les pages suivantes (voir image 2, 2014b : 23). Ces notations dans les marges des pages-écran sont fréquentes tout au long de Beauvoir, l'enquête : elles renforcent l'idée selon laquelle Frain serait une écrivaine à l'activité incessante, qui enregistre la moindre donnée pendant ses recherches, afin d'attester le caractère scientifique de son enquête.

Un exemple de la calligraphie de Frain est offert par l'image 3 (2014b : 35). L'auteure, grâce à une "métalepse interactive" (Saemmer 2007: 92), incite ses lecteurs à feuilleter les pages scannées de son carnet: "À vous de tourner les pages de mon carnet... " (Frain 2014b: 35). Dans les crédits finaux, où toutes les sources dans lesquelles elle a puisé sont listées, nous lisons, en référence à la page 35 : «pages extraites du carnet de notes d'Irène Frain ». La tentative d'offrir l'écriture manuscrite de Frain à la vue de son public se révèle ainsi encore plus audacieuse. Ce choix témoigne, en outre, d'une allusion de l'auteure à son appartenance au champ littéraire. En effet, comme le souligne Maingueneau, ce ne sont que les écrivains doués d'une auctorialité forte, ayant un Opus canonisé et un statut d'autorités, qui voient publier leurs textes qui n'étaient pas destinés à l'être, comme les brouillons, les carnets, les journaux, etc. (2013: 15-16). Bref, «l'ethos éditorial » de Beauvoir, l'enquête reflète et renforce l'« image d'auteure » de Frain, une écrivaine née dans le papier et pour le papier, mais qui vit avec son temps, qui est désireuse de passionner ses lecteurs en leur offrant des expériences littéraires nouvelles. Cet ethos montre d'ailleurs que l'éditeur lui aussi ne veut pas aller trop loin et bouleverser tous les codes séculaires du livre papier, ce qui encore une fois coïncide avec la «posture » (Meizoz 2007) de Frain. En effet, à la question « Allez-vous publier directement sur ipad certains de vos livres?", elle a répondu: "Beauvoir, l'enquête est un document, une œuvre de passeur sous format court et vivant. J'en reste pour mes romans à la même politique : publier sur support papier et support numérique simultanément » (2014a : en ligne). 


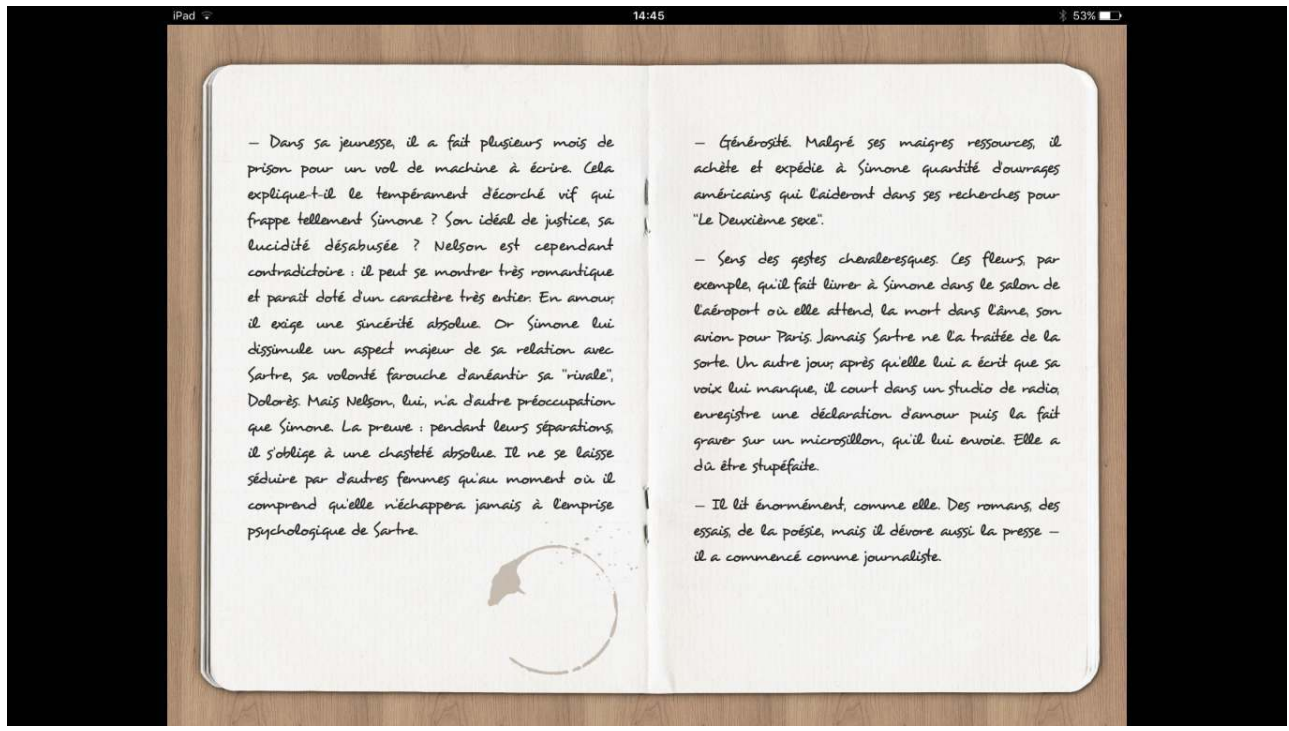

Image 1. Capture d'écran de la page 25 (Frain 2014b) faite au mois de juillet 2017. Beauvoir, l'enquête, Irène Frain, (c) Éditions Michel Lafon-Storylab, 2014

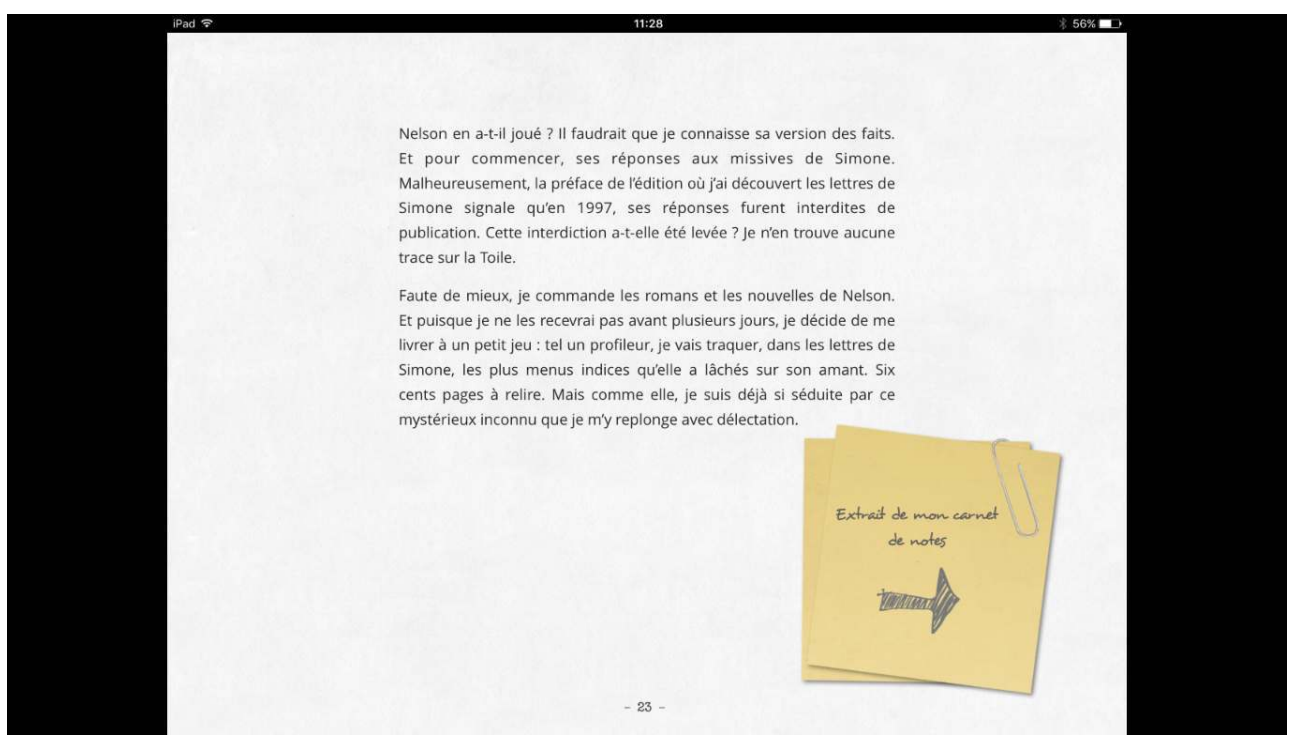

Image 2. Capture d'écran de la page 23 (Frain 2014b) faite au mois de juillet 2017.

Beauvoir, l'enquête, Irène Frain, (c) Éditions Michel Lafon-Storylab, 2014 


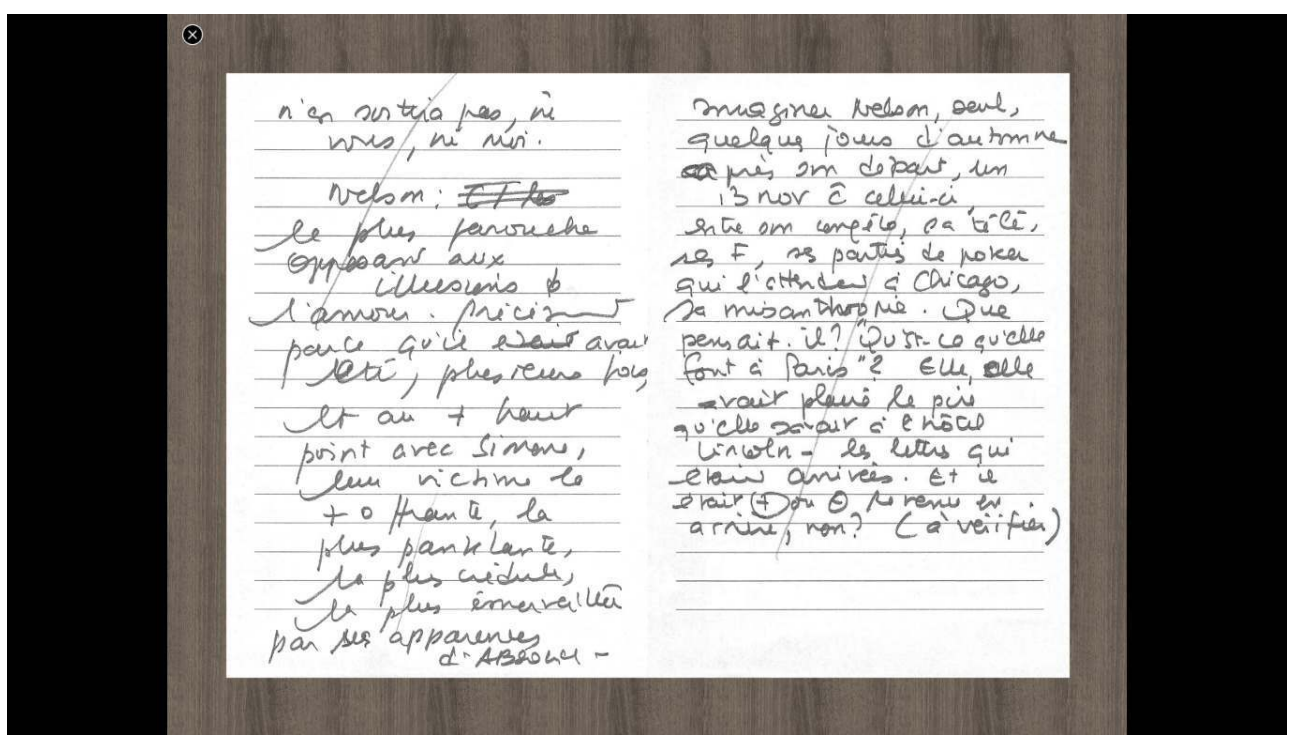

Image 3. Capture d'écran de la page 35 (Frain 2014b) faite au mois de juillet 2017.

Beauvoir, l'enquête, Irène Frain, (c) Éditions Michel Lafon-Storylab, 2014

21 Alienare, quant à lui, offre un exemple d'un «ethos éditorial» beaucoup plus expérimental, qui est tout à fait cohérent avec «l'image d'auteure » de Delaume. Son lecteur doit être prêt à s'engager dans une expérience de lecture attentive, sans craindre parfois d'être surpris, voire dérouté, par les formes d'un texte numérique qui s'invente en dehors de la tradition papier.

Tout d'abord ce texte n'a pas de couverture au sens traditionnel, il se décharge sous forme d'application sur l'App Store. En cliquant sur l'icône, le lecteur a accès à une page-écran que j'aimerais qualifier de «couverture animée » : une image mobile de la même icône est visualisée, accompagnée par une voix, une musique et des bruits créant potentiellement une ambiance inquiétante. Les noms des auteurs ainsi que le logo et le nom de la maison d'édition y sont indiqués. Il n'y a pas de frontispice, mais une autre page-écran qui suit la précédente et qui signale la date et la maison d'édition, le nom du développeur, ainsi que le fait que le Centre National du Livre a soutenu ce projet. Les pages ne sont pas numérotées et un texte défilant s'offre au lecteur, qui peut le parcourir en le scrollant verticalement. Le sommaire (voire image 4, Delaume $2015 \mathrm{c}^{6}$ ) reste toujours accessible en appuyant sur la droite de la page-écran, et constitue la seule ressource pour s'orienter dans le texte. Il propose la liste des extraits des journaux internes des personnages qui ont participé à la mission Alienare, ainsi que d'autres sections et animations qui composent le livre. 


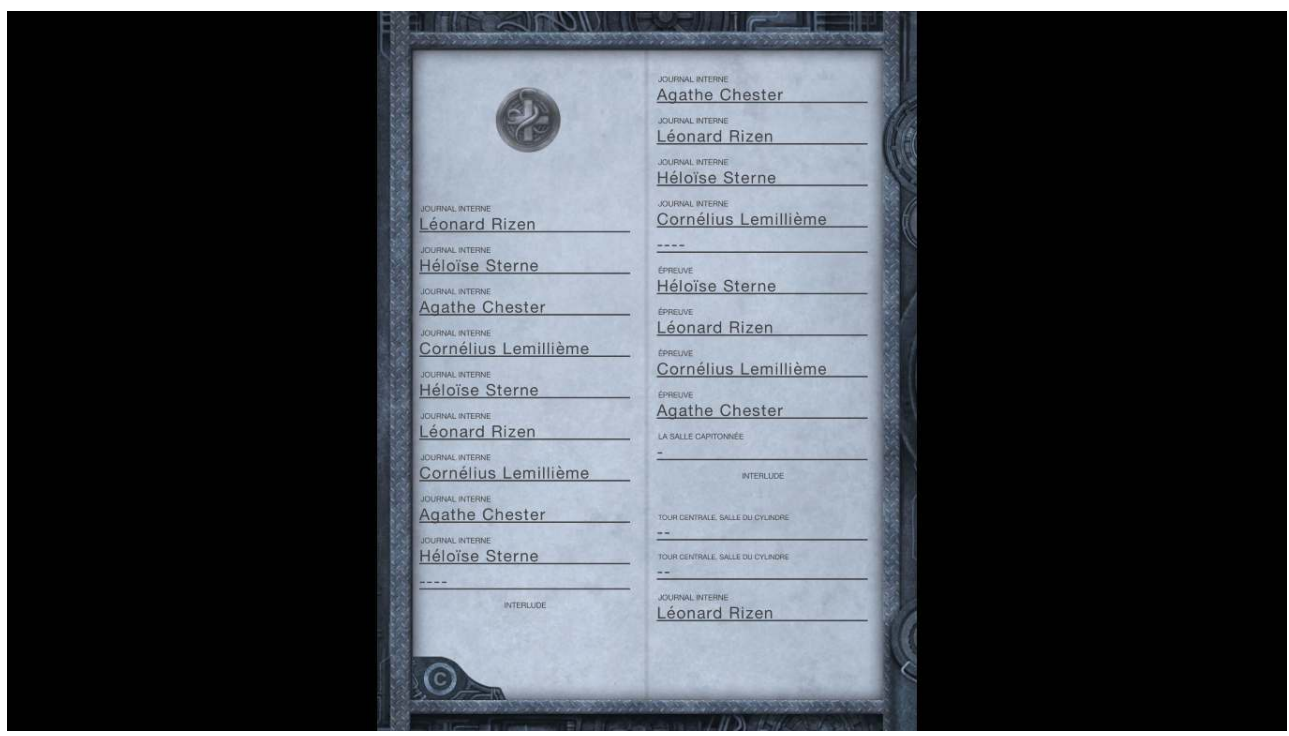

Image 4. Capture d'écran de Delaume (2015c) faite au mois de juillet 2017.

Alienare, Chloé Delaume, Franck Dion, (c) Éditions du Seuil, 2015

La forme textuelle d'Alienare est celle d'une succession de voix qui donnent vie à une performance théâtrale de type nouveau. Le journal interne de chaque personnage est associé à une police de caractère. Le lecteur est progressivement appelé à identifier la prise de parole de chaque personnage à partir du caractère typographique utilisé (voir, après le troisième extrait du journal de Cornélius Lemillième, la section intitulée «identification vocale»). Le manque de pagination, le texte défilant, les prises de parole des personnages identifiées graphiquement créent en effet une expérience potentiellement dépaysante. À cela s'ajoute le fait que la vision des caractères est parfois intentionnellement dérangée: le texte se floute, suggérant la confusion psychique des personnages (voir image 5, Delaume 2015c).
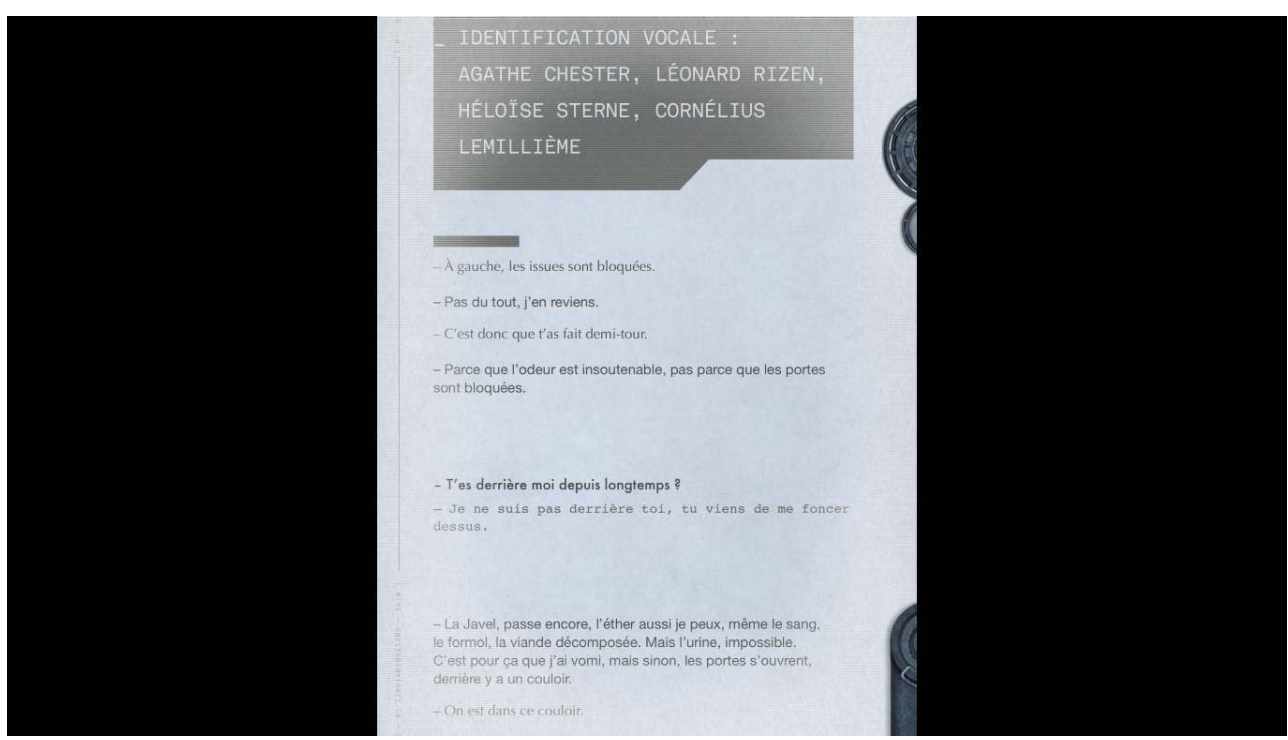

Image 5. Capture d'écran de Delaume (2015c) faite au mois de jullet 2017.

Alienare, Chloé Delaume, Franck Dion, (c) Éditions du Seuil, 2015

La couleur des pages-écran contribue à produire l'ambiance voulue par Delaume pour sa Waste Land (Eliot 1922). Alienare met en scène la maladie mentale, la mort, 
l'internement dans les asiles : c'est un monde beckettien où l'absurdité de l'existence règne, et aucun espoir n'est possible. Toutes les pages-écran et les animations jouent sur les tons de gris, ce qui donne l'idée d'une séparation par rapport à une réalité qui est, telle que beaucoup d'entre nous la connaissent, un triomphe de couleurs. Le choix de cette forme-modèle " pro-référentielle ", caractéristique selon Saemmer du discours narratif numérique, préfigure "un lecteur prêt à s'abandonner aux simulacres de référent » $(2015: 244)^{7}$, donc à se perdre parmi les voix des personnages comme dans le gris labyrinthe physique qu'ils traversent.

Les animations visuelles et sonores, qui débutent automatiquement pendant la lecture et ne peuvent pas être arrêtées, sont, quant à elles, une forme-modèle "prodepréhensive »: elles préfigurent "un lecteur acceptant de perdre prise sur l'interface » (Saemmer 2015 : 194). L'« ethos éditorial » projeté par de tels choix montre que Seuil est une maison d'édition qui nourrit un grand intérêt pour les expérimentations littéraires et les potentialités esthétiques offertes par le numérique. Un ethos aussi innovant peut sûrement séduire un lecteur habitué à la lecture immersive d'un roman papier, mais prêt à s'impliquer intensément dans l'expérimentation d'un texte à la fois lisible, visible et manipulable (et à s'y perdre parfois): un lecteur que Delaume a déjà habitué à de telles pratiques.

\section{Degrés d' "argumentativité " dans les textes du régime « élocutif » et « délocutif »}

Afin d'entrer dans le vif de l'analyse des deux livres enrichis du corpus, la distinction proposée par Maingueneau (2004) entre régime "élocutif» et "délocutif ${ }^{8}$ est intéressante. Si dans la section précédente la notion d'« image d'auteur » extratextuelle a été mise en relation avec celle d'«ethos éditorial " afin de mettre en évidence le potentiel argumentatif de cette synergie, dans cette section l'étude de l'«ethos auctorial » (Amossy 2009 : en ligne) « dit » par Frain et «montré » (Maingueneau 2004 : 206) par Delaume fait ressortir les stratégies variablement argumentatives utilisées dans Beauvoir, l'enquête et Alienare.

\subsection{Beauvoir, l'enquête : « comme dans un polar »}

Le livre enrichi de Frain est un récit autobiographique bref, qui rapporte ses recherches concernant ce qui aurait été la seule véritable histoire d'amour de Simone de Beauvoir : celle avec le poète américain Nelson Algren. Comme tout texte appartenant au régime «élocutif», la dimension de "figuration» (Maingueneau 2004 : 113) y est essentielle. Dans Beauvoir, l'enquête la "personne» de Frain est à la fois l'héroïne du récit et l'«inscripteur» du texte (Maingueneau 2004 : 113). Ce livre permet ainsi à l'écrivaine de réaffirmer la valeur artistique et la légitimité littéraire de son Beauvoir in love.

En effet, l'un des traits distinctifs de l'écriture de Frain est de prendre comme source pour ses romans des événements historiques. Du Nabab (1982) à Devi (1992), en passant par Au Royaume des Femmes (2006) ou Les Naufragés de lîle Tromelin (2009) pour arriver à Beauvoir in love (2012), les exemples foisonnent. La narration, dans ce dernier livre, se veut donc factuelle (Schaeffer 2013). L'interaction entre le réel et le fictionnel exige que le lecteur accepte le " pacte référentiel », en assumant que l'œuvre établit un lien étroit 
avec le monde réel, et que sa fiabilité peut être mise à l'épreuve sur la base de la validité des informations qui y sont données (Lejeune 1975 :36). Beauvoir, l'enquête est ainsi complémentaire et nécessaire à Beauvoir in love, étant en même temps un récit autobiographique et un dossier documentaire. Dans l'introduction animée qui ouvre ce livre enrichi (une courte vidéo où Frain s'adresse à ses lecteurs) elle affirme :

Un livre, c'est toujours l'élucidation d'un secret. [...] J'ai voulu comprendre, je suis allée sur place, à Chicago [...]. J'ai [...] noirci des carnets avant d'écrire, et ces carnets, qui ressemblent à la chronique d'un thriller, j'ai voulu les partager aujourd'hui avec vous. Vous allez voir, c'est palpitant, c'est plein de rebondissements. Des zones d'ombres se sont éclairées. On y va (2014b).

Et dans la conclusion, nous lisons :

À force de recoupements et de vérifications, j'avais réussi à faire la part des mensonges, des silences, des reconstructions après coup. J'en étais maintenant certaine : [1'] histoire [de Simone] avec Nelson fut le zénith de sa vie. [...] J'ai alors décidé de donner ma propre version de ces trois années de folie amoureuse (2014b : 70).

En se montrant physiquement à ses lecteurs dans son introduction animée, Frain évoque toute une série de représentations liées à son "image d'auteure " circulant dans le champ littéraire. C'est en tant que journaliste et chercheuse attentive et passionnée qu'elle s'adresse à son public : de façon cohérente, sa prise de parole (orale et écrite) est très marquée par sa subjectivité, comme le témoigne le recours à la première personne ainsi que l'étayage d'un champ lexical fort subjectif (voir par exemple, dans les passages susmentionnés, " palpitant », « certaine »), qui ponctue tout Beauvoir, l'enquête. La complexité plurisémiotique du livre enrichi, qui rend en même temps présentes la parole écrite de Frain, sa voix, son image, produit un mode d'énonciation nouveau qui, en exploitant plusieurs ressources multimédia, permet à Frain d'expliquer pourquoi l'histoire de Simone avec Nelson fut à ses yeux «le zénith de sa vie ».

En effet, le numérique est la possibilité pour l'auteure d'offrir à son public nombre de preuves qui confirment sa version des faits, ainsi que le bien-fondé de l'histoire qu'elle raconte dans son Beauvoir in love. Elle choisit pour son récit la "scénographie numérique " (Maingueneau 2014: 176-179) du polar, en s'inscrivant dans un genre traditionnel, tout en le renouvelant. Le titre du chapitre 2 est, en effet, « comme dans un polar ». C'est un ethos de détective de nouvelle génération que l'auteure projette tout au long de Beauvoir, l'enquête, en partageant avec ses lecteurs ses hypothèses (le titre du chapitre 4 est « Nouvelles hypothèses »), ses indices, ses pistes (voir le titre du chapitre 5), ainsi que les résultats de ses recherches.

Elle se sert de nouveaux médias pour obtenir les informations dont elle a besoin: le lecteur assiste à un échange sur Skype que Frain aurait eu avec la spécialiste des œuvres de Nelson Algren pendant qu'elle était en train de rechercher la correspondance de celui-ci avec Simone (Frain 2014b : 37-39). Il a accès aux résultats des recherches de l'écrivaine sur Internet: un grand nombre de photos (par exemple celle d'archives d'Algren, p. 21), de vidéos (comme le document INA montrant Beauvoir au travail, ainsi que la photo du couple officiel Beauvoir-Sartre, p. 11) ou d'extraits audio (comme la chanson The House of Rising Sun, qu'Algren «écoutait en boucle », p. 28). Frain insiste sur le fait que tous ces documents lui ont permis de se plonger dans l'ambiance, les sensations, les expériences vécues par Simone et Nelson, ce qui a été essentiel pour écrire son roman. 

sur les lieux des faits, à Chicago, car "lorsqu'ils enquêtent, les écrivains comme les policiers et les juges d'instruction, sont convaincus que les lieux parlent » (Frain 2014b : 42). Nombreuses sont les photos des lieux visités et elles sont présentées au moyen de ressources techniques visant à susciter un plaisir de la lecture de type nouveau. Un exemple intéressant est la carte interactive que l'on peut voir dans l'image 6 (Frain $2014 b$ : 60).

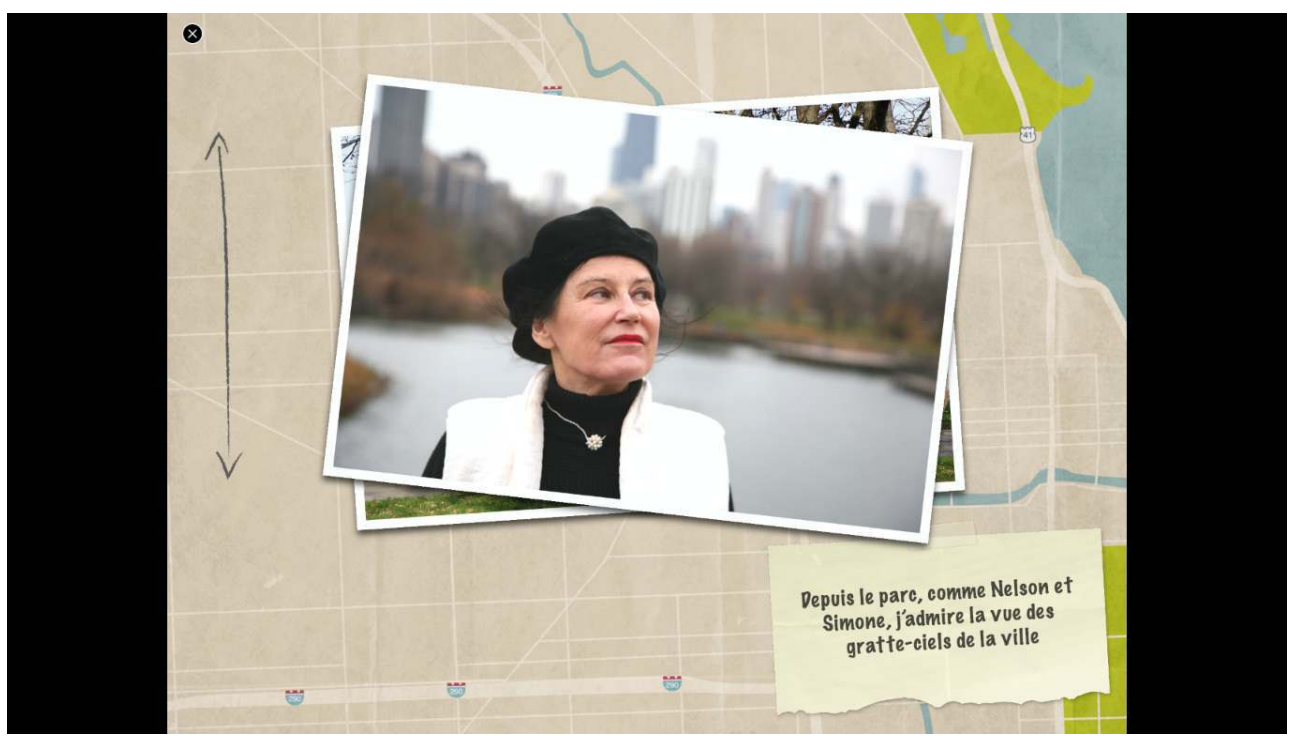

Image 6. Capture d'écran de la page 60 (Frain 2014b) faite au mois de jullet 2017

Beauvoir, l'enquête, Irène Frain, (c) Éditions Michel Lafon-Storylab, 2014

Frain multiplie également les photos prises dans les archives (voir l'image 7 - Frain $2014 \mathrm{~b}$ : 46), où elle a eu accès aux documents authentiques qui lui auraient permis de découvrir la vraie histoire des deux amants.

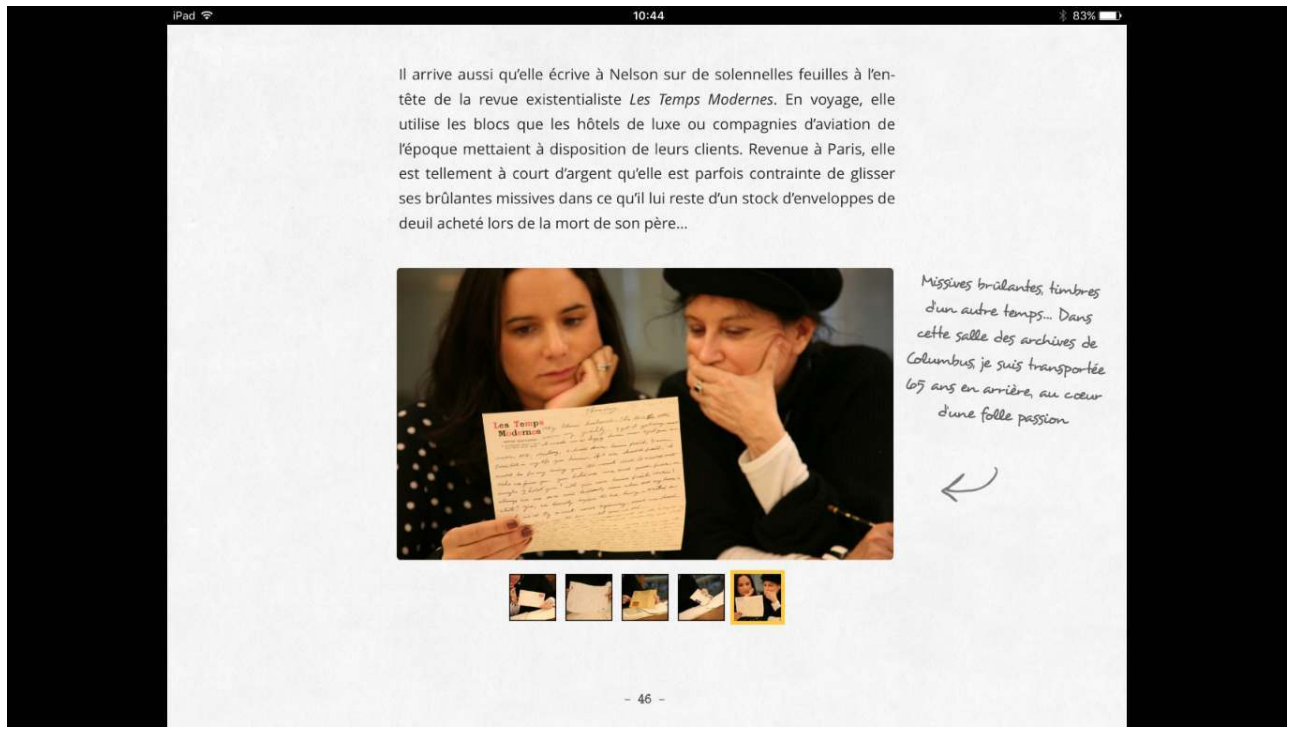

Image 7. Capture d'écran de la page 46 (Frain 2014b) faite au mois de jullet 2017.

Beauvoir, l'enquête, Irène Frain, (c) Éditions Michel Lafon-Storylab, 2014

De telles images, montrant les documents authentiques entre les mains de Frain, visent à persuader le lecteur de l'authenticité de ce récit autobiographique, ainsi que de la 
véracité de l'histoire racontée dans Beauvoir in love. Les photos sont d'ailleurs, comme l'affirme Amossy (2011:32), des preuves extra-techniques, à savoir un type de preuve qui n'a pas besoin d'être démontré, et qui agit directement au niveau du pathos. Nous sommes dans ce que la spécialiste appelle une «rhétorique du témoignage » : l'image authentique «a une présence qui lui permet d'imposer le réel plus fortement que tout discours » (Amossy $2011: 32$ ).

Les extraits de l'interview de Frain avec Art Shay (voir image 8, 2014b: 66), l'auteur de célèbres clichés volés à de Beauvoir nue dans une salle de bain, ont une fonction comparable. Il s'agit d'hyperliens de type « informationnel » qui, comme le constate Saemmer, « préfigure[nt] [...] un lecteur à la recherche de confirmations, de définitions, d'illustrations, de preuves ou d'explications» (2015: 131). Ce type d'hyperlien, qui place le lecteur dans la logique de la révélation d'un secret, et de l'apparition de vérités cachées (Saemmer 2015 : 26), est fréquent dans le discours informatif et argumentatif, notamment dans la presse en ligne.

L'«ethos auctorial» projeté dans Beauvoir, l'enquête établit un rapport étroit avec l'« image d'auteure » extratextuelle de Frain (celle de la femme de lettres, érudite et à son aise dans les archives, ainsi que de l'écrivaine passionnée et à la recherche d'une vérité à consigner à son public). Le recours à des stratégies rhétoriques " authentifiantes ", indissociables du dispositif « de monstration» (Soulages 2007) qui les véhicule, vise à donner à ce livre enrichi une dimension réaliste, et au discours de l'auteure, très marqué par sa subjectivité, un caractère d'objectivité. En effet, en assumant systématiquement le rôle d'un "sous-énonciateur" (Rabatel 2004a: en ligne), la « détective » laisse souvent " parler » ses preuves sémiotiquement variées, en essayant ainsi de fasciner un public de «lecteurs-spectateurs » de plus en plus habitué à la consultation de contenus multimédia sur le Web. Un tel mode énonciatif, dont la tablette détermine la spécificité et qui se veut en même temps "authentifiant » et "ludique », se met ainsi au service d'une "dimension argumentative» qui tend à se rapprocher de la "visée argumentative ", en mettant en évidence que les deux notions doivent être pensées comme un continuum plutôt que comme deux unités discrètes.

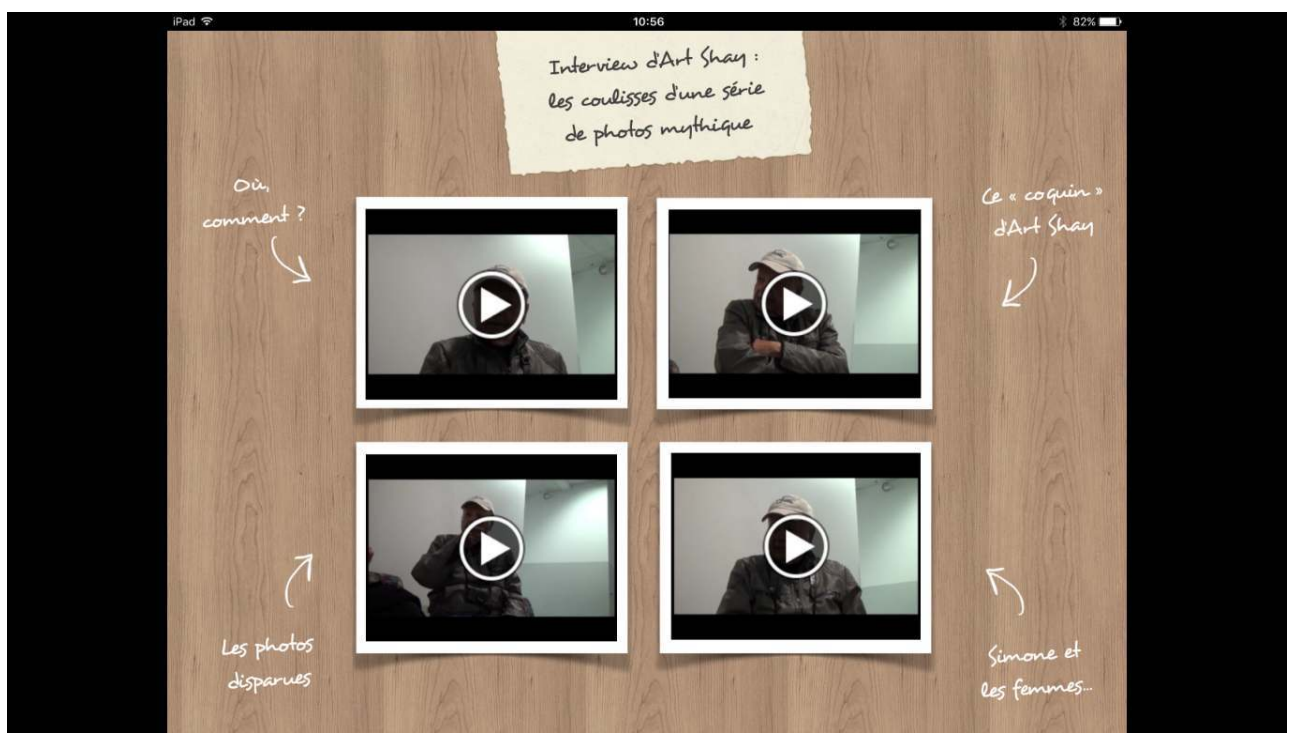

Image 8. Capture d'écran de la page 66 (Frain 2014b) faite au mois de jullet 2017.

Beauvoir, l'enquête, Irène Frain, (c Éditions Michel Lafon-Storylab, 2014 


\subsection{Alienare : le pouvoir persuasif de la peur}

110). En s'éloignant des autofictions précédentes publiées sur support papier, Delaume propose une fiction numérique qui prend la forme d'une performance expérimentale à plusieurs voix, s'inspirant des codes du cinéma d'animation et de la dramatique sonore. Dans un futur proche, six personnages (Léonard Rizen, Héloïse Sterne, Cornélius Lemilième, Agathe Chester, Major_42, Isolde von Valmut, Jean-Priam Despraves) participent à une mission dans un asile pendant quarante-huit heures: ils doivent détruire "l'unité centrale ». Le lecteur lit leurs journaux internes et a accès à leurs souvenirs, ainsi qu'à des enregistrements de voix, de bruits, à des morceaux de vidéosurveillance.

ans cette fiction, comme ailleurs dans son œuvre, Delaume se penche sur le thème de la maladie mentale. Diagnostiquée maniaco-dépressive, puis bipolaire, elle assume et proclame sa condition de malade. Les malades mentaux sont déjà les protagonistes d'œuvres telles que La Nuit je suis Buffy Summers (2007), Transhumances (2007) ou Certainement pas (2004). Ce dernier ouvrage met en scène une partie de Cluedo dans l'hôpital psychiatrique Sainte-Anne, où Delaume a été internée. L'auteure confirme la centralité de la maladie mentale dans son expérience d'écriture: "L'internement apprend au Je à s'écrire autrement, à contrer les ficelles de sa pathologie. [...] S'écrire durant un internement influe sur la structure de Je, qui, en ces instants, est friable, éclaté » (2008 : en ligne). La maladie mentale, causée par le drame familial, est d'ailleurs à l'origine de l'expérience d'écriture de Delaume, avatar moderne du " poète-mourant » romantique selon Ruiz (2015:252), et représente un trait distinctif de son "image d'auteure ». Une telle condition "paratopique" (Maingueneau 2004: 72) s'incarne volontiers dans des personnages féminins ayant une identité fragmentée, ou habités par la folie, et dans des lieux «paratopiques» (Maingueneau 2004 : 102) tels que les asiles.

40 Si tous les personnages d'Alienare ont des traits "paratopiques » (Maingueneau 2004 : 86), l'embrayeur «paratopique » principal est Héloïse Sterne. Elle est psychiatre, elle connaît donc très bien les maladies mentales ainsi que leurs traitements : elle a rédigé le Grand Manuel des Classifications des Troubles Mentaux et elle a inventé des médicaments pour tout. Son travail consiste à "optimiser l'humain" par la psychiatrie (Delaume 2015c). En lisant son journal interne, nous découvrons cependant qu'Héloïse est ellemême malade, on pourrait dire schizophrène, vu le dialogue constant qui a lieu entre les différentes parties de son moi fragmenté. L'« ethos auctorial » projeté est celui d'une écrivaine qui connaît très bien la maladie mentale, ainsi que la psychiatrie et les psychotropes. Tout comme la création littéraire, les médicaments l'ont aidée à faire face à son drame familial. La « magie » de l'autofiction lui a consenti de corriger un réel absurde et douloureux, elle le répète d'ailleurs souvent dans ses écrits. À l'instar de Delaume, Héloïse Sterne est une "sorcière » de la pharmacologie, ce qui lui permet « d'optimiser le réel». Ainsi l'ethos que la voix de ce personnage projette, reflète à la fois la condition "paratopique » de l'auteure et s'inscrit dans son projet autofictif de longue haleine.

41 L'ethos « montré » par Delaume dans son livre enrichi confirme la littérarité d'Alienare. Le lecteur de ses romans y retrouve tous les traits stylistiques essentiels de son écriture : de l'utilisation de phrases courtes, elliptiques, hachées, à l'usage inhabituel et 
déroutant de la ponctuation; de la présence de formules mathématiques incompréhensibles dans le corps du texte à celle de néologismes et de mots inusités. La recherche d'une prose poétique intensément rythmique, riche en assonances et en consonances, est un autre indice de la volonté de l'auteure de s'inscrire dans une longue tradition littéraire et poétique. Dans quelques extraits des journaux internes, notamment dans celui de Léonard Rizen, le lecteur reconnaît des vers, souvent des alexandrins blancs, divisés en deux hémistiches, non rejetés à l'alinéa. Le rythme régulier, presque une cantilène, qui caractérise les prises de parole de ce personnage tend à renforcer l'effet d'aliénation qui résume sa condition: Rizen (un cyborg) se révèle être un véritable esclave de nos sociétés productivistes et de l'hyperconsommation. Il sera par ailleurs le « dernier survivant » à la mission Alienare, le seul qu'une telle société, et son produit le plus affreux pour Delaume, les asiles, n'ont pu tuer.

Contrairement à celle de Frain, la prise de parole de Delaume dans son livre enrichi se veut objectivante. L'auteure n'exprime pas directement ce qu'elle pense des hôpitaux psychiatriques, en gommant toute trace de sa subjectivité dans son discours littéraire. Elle se cache derrière ses personnages, en jouant sur l'« effacement énonciatif » (Rabatel 2004b), et se limite à rapporter tout ce que chacun d'eux dit, fait, pense ou éprouve. Comme dans un montage filmique elle enregistre tous les événements qui les conduisent à leur mort, une mort inéluctable et absurde, dont le seul responsable est le lieu dystopique où la mission se déroule.

L'«effacement énonciatif» produit ainsi ce que Rabatel appelle un «effacement argumentatif» (2004b: en ligne) essentiel pour la construction d'une «dimension argumentative " visant à influencer l'opinion du public. Grâce à un tel effacement, «les états du monde dénotés (valeur descriptive) se doublent d'une valeur argumentative (interprétative) à valeur d'argument, sur le mode des évidences perceptuelles ou conceptuelles » (2004b : en ligne). Une telle argumentation indirecte est d'autant plus efficace qu'elle est difficilement réfutable, les faits étant présentés comme des évidences. La démarche argumentative de Delaume, dont la position personnelle sur la légitimité de l'existence des asiles reste implicite, tend donc à montrer plutôt qu'à démontrer. Dans ce cadre, un rôle essentiel est joué par un support tel que la tablette, dont Delaume sait bien exploiter la complexité sémiotique. Son mode énonciatif, à la fois «fictif» et « ludique », fait appel à la totalité des sens de ses « lecteurs-spectateurs », en exploitant tout un éventail de ressources sémiotiques pour susciter en eux des réactions et des émotions susceptibles d'orienter leur opinion. La spécificité de la "scénographie numérique» (Maingueneau 2014:176-179) d' Alienare, associant le texte à des extraits audio et à des animations vidéo, produit une atmosphère potentiellement angoissante pour le lecteur, qui fait écho à ce qui est raconté dans le récit. Delaume avait d'ailleurs déclaré avoir « envie d'écrire un roman qui se passerait dans un futur post-apocalyptique, [...] [et de] réussir à créer de l'angoisse, de la peur » (Delaume 2012 : en ligne). Elle a récemment ajouté que, dans la réalisation d'Alienare, "Stalker [a été] une référence capitale. Mais [...] aussi [les] décors et [...] l'atmosphère de Shutter Island de Scorsese, [...] [le] labyrinthe de Shining " (2015b : en ligne).

45 Un exemple intéressant de cet « effacement argumentatif » qui mobilise des ressources plurisémiotiques est offert par les Dix Commandements de l'hôpital psychiatrique (voir le premier "Interlude»), une réécriture en version antiphrastique de ceux de la 
tradition chrétienne. Voici, à titre d'exemple, le quatrième : « Tu dormiras six jours [...]. Mais le septième jour est le jour des ateliers thérapeutiques : [...] tu sculpteras dans la glaise ta voisine schizophrène obèse, partageras ton tapis de yoga avec l'autiste incontinent du secteur 13 , et ta petite caméra avec l'anorexique psychotique du moment " (Delaume 2015c). La lecture de ces commandements est accompagnée par une musique inquiétante et par deux voix, l'une masculine, l'autre féminine, qui mettent en scène un dialogue suggestif entre un homme, qui semble être le chef de service de l'hôpital, et une patiente.

Dans Alienare la musique, les bruits, les voix lisant les passages les plus troublants ou reproduisant les cris et les délires des affolés veulent éveiller un imaginaire perceptif et cognitif qui est présent chez tout un chacun. Les traitements parfois très problématiques dans les asiles font désormais partie d'un imaginaire collectif, dans lequel le monde littéraire et cinématographique a déjà abondamment puisé. Delaume dénonce ainsi implicitement leur existence en misant sur la valeur argumentative des éléments doxiques (Amossy 2010a: 94), sur un savoir partagé depuis longtemps en circulation dans l'interdiscours.

Un autre exemple intéressant est le passage qui raconte l'histoire de la création des hôpitaux psychiatriques (dans la section appelée «La salle capitonnée »), lu par une voix masculine et une voix féminine qu'on dirait celle de Delaume. Les deux voix parfois se répondent, parfois se superposent ou s'élèvent à l'unisson. En voici un extrait :

Il était tant de fois un royaume endormi. Un royaume où la loi cousait chaque paupière, et où les bouches souriaient d'être soumises à la nuit. [...] Souffrir devint un crime. [...] Le profit devint maître des corps et des cerveaux [...] Le Styx en déborda, et à cette époque furent créées les écluses [...]. Le peuple des affolés fut muré, peu à peu. Leurs plaintes, derrière le plâtre, égrenaient les fissures (Delaume 2015c).

La lecture à voix haute de tout l'extrait est accompagnée par une animation (voir image 9, Delaume 2015c). Celle-ci montre une cellule épouvantable, en essayant d'orienter l'imagination du lecteur et de lui faire croire que les patients étaient "emprisonnés " dans des immeubles délabrés et insalubres de ce genre. Sont ainsi potentiellement mobilisées chez le public la pitié et l'indignation, deux sentiments moraux reliés à la notion d'injustice et ayant un fondement rationnel (Amossy 2010a : 168). Delaume fait ainsi appel à un pathos "à l'état pur ", comme le dirait Amossy, car "le discours qui soulève l'indignation est dépourvu de marques d'affectivité. Moins l'émotion s'inscrit verbalement, plus fortement elle semble pouvoir être suscitée chez le lecteur» (Amossy 2004 : en ligne). 


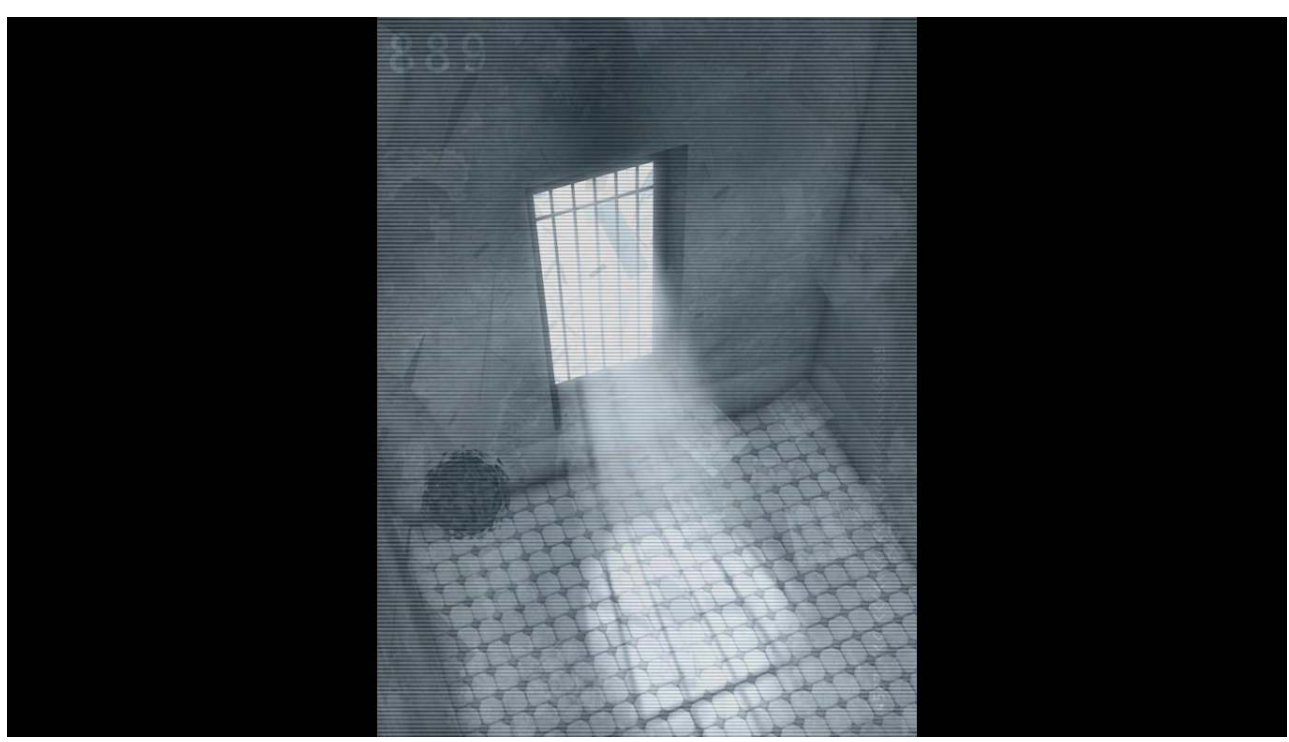

Image 9. Capture d'écran de Delaume (2015c) faite au mois de juillet 2017.

Alienare, Chloé Delaume, Franck Dion, (c) Éditions du Seuil, 2015

49 L'image 9 est pour sa part un véritable argument visuel de nature pragmatique (Roque 2008 : 185), qui dénonce l'existence des asiles en raison des conséquences mortelles que leur ouverture a entraînées. Le public est ainsi invité à tirer des conclusions de faits qui sont présentés comme des évidences, et à prendre position. L'association entre le récit et de telles animations, nombreuses dans ce livre enrichi, vise à agir sur le pathos sans pour autant négliger la dimension rationnelle : cela confirme que « visual rhetoric can be simultaneously emotional and rational seeking to persuade the viewer by means both of pathos and of a pragmatic argument » (Roque 2008 : 186).

50 J'ai également relevé dans les vidéos d'Alienare le recours à différents types de « figures d'animation ", notion par laquelle Saemmer désigne " une relation - entre des textes ou images et un mouvement - dans laquelle la sémiose est basée sur des processus d'intersection de traits signifiants associés au mouvement, au texte/à l'image, et aux contextes» (2011 : en ligne). Par traits signifiants la spécialiste en S.I.C. entend « les caractéristiques que le "lecteur modèle" [...] associe à un mouvement parce qu'elles ont été sélectionnées au long des expériences répétées dans une culture » (2011 : en ligne).

51 Ainsi, l'animation qui suit l'« épreuve de Léonard Rizen ", qui se trouve prisonnier de la pharmacie de l'hôpital psychiatrique (voir image 10, Delaume 2015c) et est forcé à avaler une grande quantité de médicaments, offre un exemple de l'« unité sémiotique de mouvement » appelée par Saemmer l'« obsessionnel» (2011: en ligne). Nombre de médicaments défilent sur des étagères, allumés par un néon clignotant très rapidement. Les «traits signifiants» que ce clignotement caractéristique de l'« obsessionnel » tend à activer, sont l'urgence et le stress. L'animation réalisée par Dion renforce ainsi l'ambiance de nervosité et la sensation de danger. Celle-ci est intensifiée par les sons, les notes brèves et frénétiques du violon ainsi que par les voix, qui dressent de façon pressante une longue liste de maladies mentales et de médicaments. Dion avoue avoir recherché précisément cet effet : «Du témoignage de personnes disparues à la topologie même des lieux que le lecteur découvre en avançant dans le récit, la hantise est omniprésente " (dans Delaume 2015b: en ligne). Dans l'animation suivante (image 11, Delaume 2015c), le lecteur voit une porte fermée dans l'un des couloirs de l'asile, et entend plusieurs voix venant de l'intérieur, parmi 
lesquelles on distingue clairement quelqu'un crier «I want to get out ». L'idée suggérée par une telle succession de séquences animées est que les psychotropes créent une addiction chez les patients, dont il est impossible de se libérer. Le lecteur ne peut qu'éprouver de l'empathie, de l'angoisse et de la peur pour la destinée de ces affolés, des émotions ayant un caractère persuasif indiscutable (Amossy 2010a : 164).

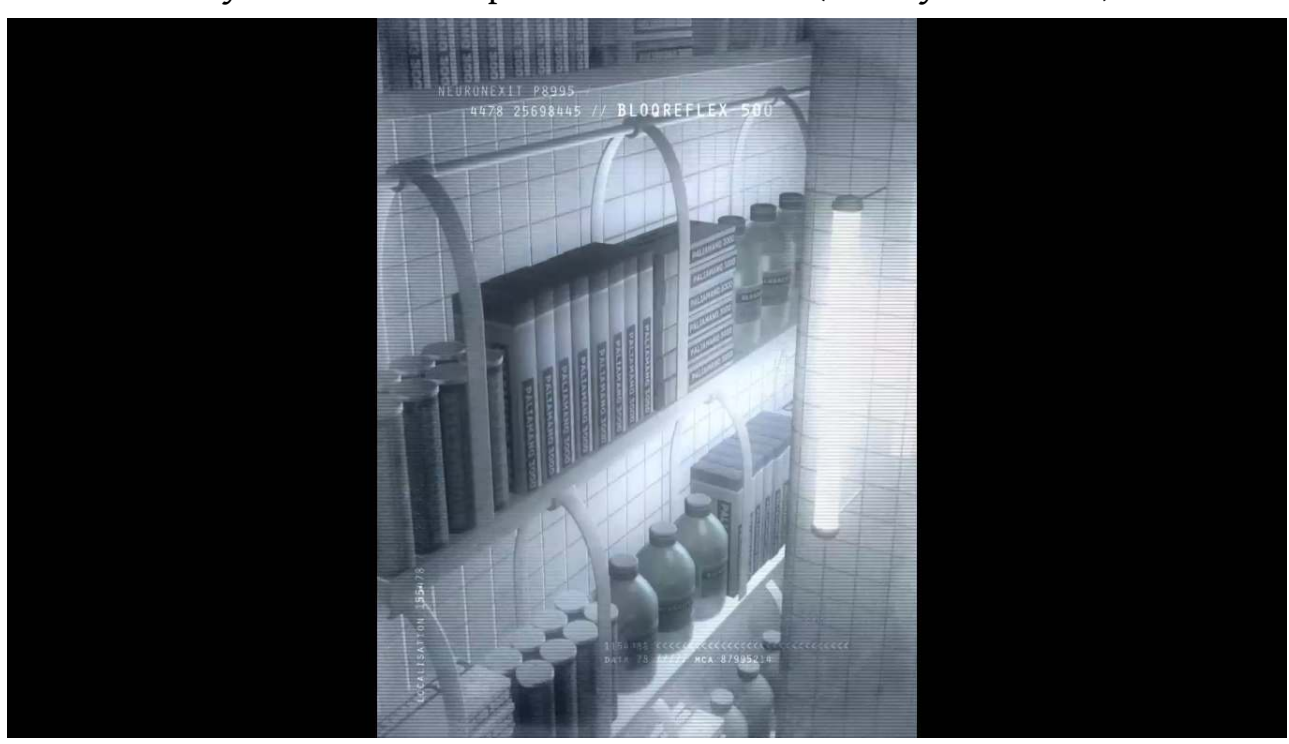

Image 10. Capture d'écran de Delaume (2015c) faite au mois de juillet 2017.

Alienare, Chloé Delaume, Franck Dion, (c) Éditions du Seuil, 2015

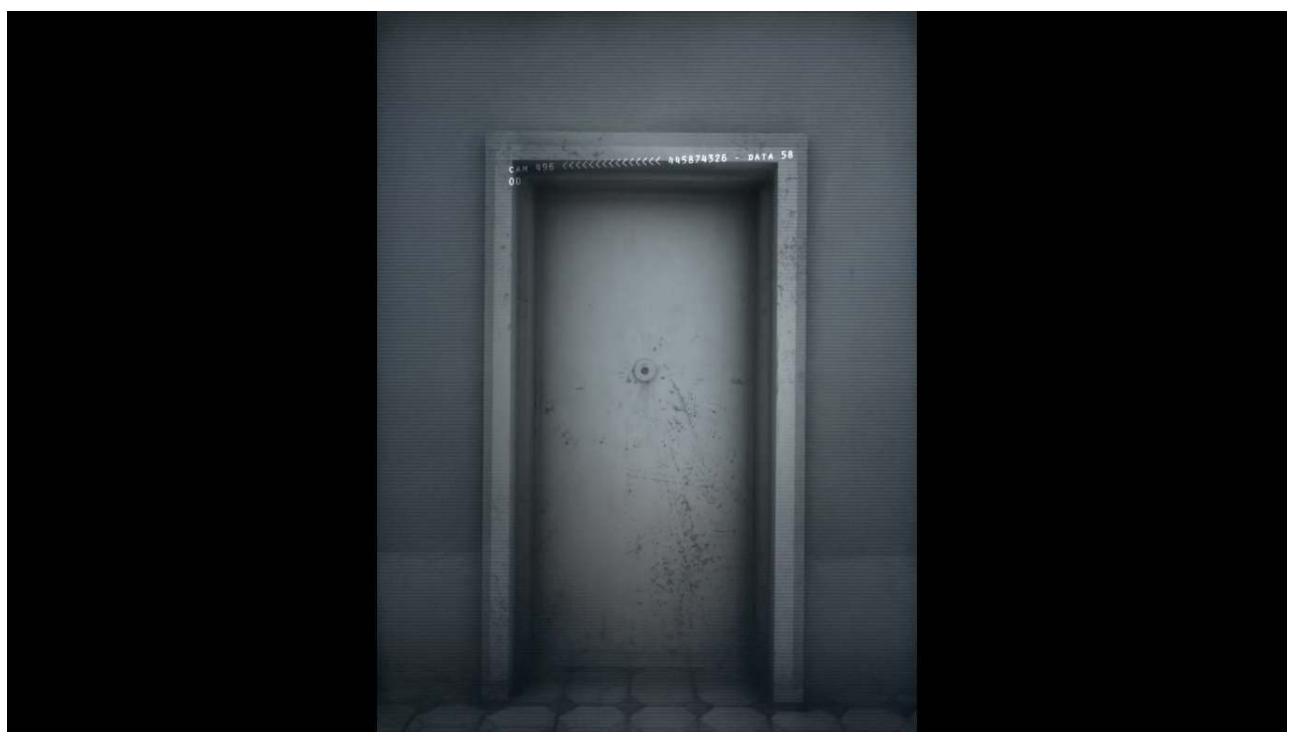

Image 11. Capture d'écran de Delaume (2015c) faite au mois de juillet 2017.

Alienare, Chloé Delaume, Franck Dion, (c) Éditions du Seuil, 2015

Le recours à de telles «figures d'animation » est une confirmation supplémentaire que l'«ethos auctorial» projeté par Delaume dans Alienare est celui de l'écrivaine qui s'engage profondément dans l'expérimentation, en essayant d'élargir les terrains sémantiques et sémiotiques de l'œuvre littéraire. Texte, sons, musique et animations métamorphosent l'écran, qui devient un espace d'immersion onirique, dans lequel une nouvelle forme de performance numérique se joue : un ensemble signifiant où plusieurs sens sont simultanément stimulés, et dans lequel le corps du lecteur est profondément impliqué (comme au théâtre ou dans la poésie orale, Zumthor 1983 : 156). Dans une telle 
performance numérique, la «dimension argumentative » n'est pas bâtie seulement au niveau textuel, mais également grâce à la synergie que créent le texte écrit, les animations, la musique, les bruits, les voix. Toute l'ampleur et la fécondité de la notion devient ainsi explicite lorsqu'elle est mise en relation avec les potentialités plurisémiotiques du multimédia.

\section{Conclusion}

Cette étude, en s'inscrivant dans la réflexion d'Amossy qui insiste sur la nécessité d'«embrasser dans le même champ d'étude toutes les modalités selon lesquelles la parole tente d'agir dans l'espace social » (2010a: 7), a montré, je l'espère, que l'image d'une auteure qui circule dans le champ littéraire a une capacité remarquable d'orienter la réception des œuvres. Je me suis d'abord penchée sur "l'image d'auteure " extratextuelle de Frain et de Delaume, en montrant qu'une première dimension d'« argumentativité » de leurs livres enrichis se définit au niveau para-péritextuel, grâce à l'association qui se crée entre leurs " images d'auteures » et l'« ethos éditorial » des deux œuvres.

En passant à l'étude de l'« image d'auteure » intratextuelle et en différenciant l'« ethos auctorial» «dit» par Frain et "montré» par Delaume, j'ai examiné le degré d'objectivité et de subjectivité des prises de parole des deux auteures pour voir comment il se met au service de la construction de la «dimension argumentative » de Beauvoir, l'enquête et d'Alienare. L'ethos de détective de Frain et la "rhétorique du témoignage " à laquelle elle a recours, en exploitant abondamment les ressources multimédia, produisent une synergie profonde entre « image d'auteure » extratextuelle et "ethos auctorial», visant à persuader le lecteur de la véracité de sa version de l'histoire d'amour racontée dans Beauvoir in love. Delaume privilégie, quant à elle, une prise de parole objectivante dans son Alienare, qui favorise la construction d'une "dimension argumentative» par le recours à l'implicite et à l'«effacement énonciatif ». La matérialité discursive plurisémiotique des deux livres enrichis se met, dans chaque cas de façon spécifique, au service de la démarche argumentative des deux auteures, qui peut être plus ou moins explicitement argumentative au niveau textuel, mais qui est en revanche dans les deux cas subrepticement argumentative au niveau para-péritextuel.

L'analyse du corpus a enfin mis en évidence que l'«argumentativité » de ces textes littéraires est strictement liée à un travail attentif des auteures sur le pathos du public. L'intrication entre dimension rationnelle et pathémique est essentielle : logos et pathos sont synergiques. Associés aux ethè auctoriaux respectifs des deux écrivaines, les trois dimensions orientent potentiellement l'expérience de lecture du public et essaient de guider sa vision des choses, tout en le tenant sous le charme d'une expérience esthétique de type nouveau. 


\section{BIBLIOGRAPHIE}

Amossy, Ruth. 2011. « La rhétorique des photos de guerre dans les polémiques électroniques. L'exemple de a@si », Migliore, Tiziana (éd.). Retorica des visibile. Strategie dell'immagine tra significazione e comunicazione (Roma : Aracne), 25-40

Amossy, Ruth. 2010a. L'argumentation dans le discours (Paris : Colin)

Amossy, Ruth. 2010b. La présentation de soi : ethos et identité verbale (Paris : PUF)

Amossy, Ruth. 2009. « La double nature de l'image d'auteur ", Argumentation et Analyse du Discours 3, en ligne : http://aad.revues.org/662

Amossy, Ruth. 2004. «L'espèce humaine de Robert Antelme ou les modalités argumentatives du discours testimonial », Semen 17, en ligne : http://journals.openedition.org/semen/2362

Amossy, Ruth, Roselyne, Koren. 2004. « Présentation », Semen 17, en ligne : http:// journals.openedition.org/semen/2305

Bouchardon, Serge. 2014. La valeur heuristique de la littérature numérique (Paris : Hermann)

Bouchardon, Serge. 2005. Le récit littéraire interactif. Narrativité et interactivité. Thèse de doctorat, Université de Technologie de Compiègne (décembre 2005)

Delaume, Chloé. 2015a. « Alienare, un livre-film qui se lit comme une appli », propos recueillis par Claire Richard, Rue89 8, en ligne : http://tempsreel.nouvelobs.com/rue89/rue89-rue89-culture/ 20151108.RUE1222/alienare-un-livre-film-qui-se-lit-comme-une-appli.html

Delaume, Chloé. 2015b. «Interview 2015 : Chloé Delaume et Franck Dion pour Alienare », entretien avec Jean-Laurent Del Socorro, ActuSF.com, en ligne : http://www.actusf.com/spip/ Interview-2015-Chloe-Delaume-et.html

Delaume, Chloé. 2012. «Le Soi est une fiction », Chloé Delaume s'entretient avec Barbara Havercroft, Revue Critique de Fixxion Française Contemporaine/Critical Review of Contemporary French Fixxion 4, 126-136, en ligne : http://www.revue-critique-de-fixxion-francaise-contemporaine.org/ rcffc/article/view/fx04.12/670

Delaume, Chloé. 2009. « Chloé Delaume : "Le numérique est une ouverture sur la littérature” », propos recueillis par Camille Tenneson, Bibliobs.com, en ligne : http://bibliobs.nouvelobs.com/ actualites/20090424.BIB3298/chloe-delaume-le-numerique-est-une-ouverture-sur-lalitterature.html

Delaume Chloé. 2008. « S'écrire mode d'emploi », intervention au colloque de Cerisy sur l'autofiction, 25 juillet, (éd. complète : publie.net), en ligne : http://classes.bnf.fr/ecrirelaville/ ressources/delaume.pdf

Ducas, Sylvie. 2010. «Fiction auctoriale, postures et impostures médiatiques : le cas de Chloé Delaume, "personnage de fiction" ", Le Temps des médias 14, 176-192

Eliot, Thomas Steams. 1922. The Waste Land, (New York : Horace Liveright)

Frain, Irène. 2014a. «Irène Frain et l'aventure numérique Beauvoir, l'enquête », entretien avec Emmanuelle de Boysson, Bscnews.fr, en ligne : https://bscnews.fr/201405253820/Point-de-vue/ irene-frain-et-l-aventure-numerique-beauvoir-l-enquete.html

Grégoire, Monique. 1993. « Irène Frain : Les mots nus du désir », Nuit blanche 53, 64-67 
Iser, Wolfgang. 1995. L'acte de lecture. Théorie de l'effet esthétique (Bruxelles : Mardaga)

Jauss, Hans R. 2010. Pour une esthétique de la réception (Paris : Gallimard)

Lejeune, Philippe. 1975. Le Pacte Autobiographique (Paris : Seuil)

Jeanneret, Yves, Emmanuel, Souchier. 2005. «L'énonciation éditoriale dans les écrits d'écran », Communication et langages 145, 3-15

Maingueneau, Dominique. 2014. Discours et analyse du discours (Paris : Colin)

Maingueneau, Dominique. 2013. «Écrivain et image d'auteur », Maingueneau, Dominique, Pascale, Delormas \& Inger, Østenstad (éds.). Se dire écrivain. Pratiques discursives de la mise en scène de soi (Limoges : Lambert-Lucas), 13-28

Maingueneau, Dominique. 2009. « Auteur et image d'auteur en analyse du discours », Argumentation et Analyse du Discours 3, en ligne : http://aad.revues.org/660

Maingueneau, Dominique. 2004. Le Discours littéraire. Paratopie et scène d'énonciation (Paris : Colin) Perelman, Chaïm, Lucie, Olbrechts-Tyteca. 2000. Traité de l'argumentation (Bruxelles : Éditions de l'Université de Bruxelles)

Meizoz, Jérôme. 2007. Postures littéraires. Mises en scène modernes de l'auteur (Genève : Slaktine)

Rabatel, Alain. 2004a. «L'effacement énonciatif dans les discours rapportés et ses effets pragmatiques », Langages 156, en ligne : http://www.persee.fr/web/revues/home/prescript/ article/lgge_0458-726x_2004_num_38_156_960

Rabatel, Alain. 2004b. «Effacement énonciatif et effets argumentatifs indirects dans l'incipit du Mort qu'il faut de Semprun ", Semen 17, en ligne : http://journals.openedition.org/semen/2334

Roque, Georges. 2011. « Rhétorique visuelle et argumentation visuelle », Semen 32, en ligne : http://semen.revues.org/9370

Roque, Georges. 2008. « Political Rhetoric in Visual Images », Weigand, Edda (éd.). Dialogue and Rhetoric (Amsterdam/Philadelphia : John Benjamins Publishing Company), 185-193

Ruiz, Ugo. 2015. Le blog d'écrivain : La littérature à l'épreuve d'Internet, thèse en cotutelle pour obtenir le grade de docteur de l'Université Paris-Sorbonne et de l'Université de Göteborg, sous la codirection de Dominique Maingueneau et Richard Sörman, soutenue en février 2015

Saemmer, Alexandra. 2015. Rhétorique du texte numérique (Villeurbanne : Presses de l'Enssib).

Saemmer, Alexandra. 2011. «De la confirmation à la subversion: les figures d'animation face aux conventions du discours numérique », Protée 39 : 1, 23-36, en ligne : http://www.erudit.org/ revue/pr/2011/v39/n1/1006724ar.html?vue=integral

Saemmer, Alexandra. 2007. Matières textuelles sur support numérique (Saint-Étienne : Presses Universitaires de Saint-Étienne)

Saemmer, Alexandra, Nolwenn, Tréhondart. 2014. « Les figures du livre numérique "augmenté" au prisme d'une rhétorique de la réception ", Études de communication 43, 107-128

Schaeffer, Jean-Marie. 2013. « Fictional vs Factual Narration », Hühn, Peter \& al. (éds.). The living Handbook of Narratology (Hamburg : Hamburg University Press), online : http://wikis.sub.unihamburg.de/lhn/index.php/Fictional_vs._Factual_Narration

Soulages, Claude. 2007. Les rhétoriques télévisuelles. Le formatage du regard (Bruxelles : Éditions De Boeck) 
Tréhondart, Nolwenn. 2014. « Le livre numérique "augmenté" au regard du livre imprimé : positions d'acteurs et modélisations de pratiques ", Les Enjeux de l'information et de la communication 15 : 2, 23-37, en ligne : www.cain.info/revue-les-enjeux-de-l-information-et-de-lacommunication-2014-2-page-23.htm

Tréhondart, Nolwenn. 2013. « Le livre "enrichi”, un hypermédia en construction : Enquête sur les représentations des éditeurs", Saleh, Imad, Manuel, Zacklad (éds.), Pratiques et usages numériques H2PTM'13 (Paris : Hermès-Lavoisier), 175-189

Zumthor, Paul. 1983. Introduction à la poésie orale (Paris : Seuil)

\section{Corpus étudié}

Delaume, Chloé. 2015c. Alienare, avec Franck Dion et Sophie Couronne, coll. « Romans français » (Paris : Seuil) [Disponible uniquement sur iPad]

Frain, Irène. 2014b. Beauvoir, l'enquête, édition Storylab (en coédition avec Lafon), coll. « Essais \& Documents » [Disponible uniquement sur iPad]

Frain, Irène. 2012. Beauvoir in love (Neuilly-sur-Seine : Lafon)

\section{Émissions de radio ou de télévision}

Frain, Irène. s. d. « Entretien avec Irène Frain » de Nathalie de Broc - émission de radio 2 De Bric et de Broc, en ligne : http://www.nathaliedebroc.fr/index.php/emissions-de-radio2

Frain, Irène. s. d. Rencontre avec l'écrivaine animée par Hubert Prolongeau au Musée du quai Branly-Jacques Chirac, en ligne : https://www.franceculture.fr/conferences/musee-du-quaibranly-jacques-chirac/irene-frain-rencontre-animee-par-hubert-prolongeau? xtmc $=i$ \%C $3 \%$ A 8 ne\%20frain\&xtnp $=1 \&$ xtcr $=2$

\section{NOTES}

1. Une définition officielle du livre enrichi a été proposée par la loi n 2011-590 du 26 mai 2011 et par le décret qui précise les caractéristiques des livres entrant dans le champ d'application de cette loi.

2. Le livre enrichi peut accueillir d'autres formes discursives, telles que les catalogues d'exposition pour tablette ou les livres de cuisine enrichis pour téléphone portable, que je ne prendrai pourtant pas en compte ici.

3. Je tiens à remecier, pour leur aimable autorisation de reproduction des captures d'écran des deux livres enrichis, les auteurs Irène Frain, Chloé Delaume, Franck Dion, et les éditeurs Michel Lafon-Storylab et Seuil.

4. Elle le résume parfaitement dans ce passage, qui débute par l'embrayeur d'autofiction récurrent dans ses romans : «Je m'appelle Chloé Delaume. Je suis un personnage de fiction. Je le dis, le redis, sans cesse partout l'affirme. [...] J'utilise, comme mes pairs, le vécu comme matériau. [...] S'écrire, non pas à nu, mais parfaitement à vif » (Delaume 2008 : en ligne).

5. «L'énonciation éditoriale [...] désigne l'ensemble de ce qui contribue à la production matérielle des formes qui donnent au texte sa consistance, son "image de texte". Il s'agit d'un processus social déterminé, [...] qui peut [...] être appréhendé à travers la marque qu'impriment les pratiques de métiers constitutives de l'élaboration, de la constitution ou de la circulation des textes. [...] Plus fondamentalement, l'énonciation éditoriale est ce par quoi le texte peut exister matériellement, socialement, culturellement... aux yeux du lecteur» (Jeanneret et Souchier $2005: 6)$. 
6. Étant donné le manque de pagination, toute référence à ce livre enrichi se limitera à signaler la date de sa publication.

7. Le texte numérique est capable de devenir simulacre de référent dans la mesure où la couleur, les unités sémiotiques temporelles ou de la manipulation qui le caractérisent, peuvent renvoyer par leurs caractéristiques sensibles à des référents d'expérience (Saemmer $2015: 244$ ).

8. Comme l'explique Maingueneau, «la littérature entremêle deux régimes : un régime qu'on pourrait dire délocutif dans lequel l'auteur s'efface devant les mondes qu'il instaure, et un régime élocutif, dans lequel "l'inscripteur", "l'écrivain" et la "personne", conjointement mobilisés, glissent l'un sur l'autre » (2004:110).

\section{RÉSUMÉS}

Le corpus de cette étude se compose de deux livres enrichis : Beauvoir, l'enquête, par Irène Frain, et Alienare, par Chloé Delaume. En puisant dans la réflexion de la rhétorique du texte numérique, j'analyse le rapport entre l'« image d'auteure » de Frain et de Delaume et l'« ethos éditorial » des deux livres, en montrant que les maisons d'édition ont essayé d'orienter la réception de ces œuvres et que leur "dimension argumentative" se manifeste d'abord à un niveau parapéritextuel. En croisant la réflexion proposée par l'analyse du discours littéraire avec l'approche développée par l'argumentation dans le discours, j'étudie les « ethè auctoriaux " projetés par les deux écrivaines dans leurs œuvres. Le niveau d'objectivité/subjectivité de leurs discours, ainsi que les stratégies rhétoriques mobilisées, ont été pris en compte pour réfléchir sur le caractère complexe et graduel de la notion de « dimension argumentative », lorsqu'elle est mise à l'épreuve des potentialités plurisémiotiques du multimédia.

The case study of this paper are two enhanced books: Beauvoir, l'enquête, by Irène Frain, and Alienare, by Chloé Delaume. The rhetoric of the digital text is essential to the study of the relationship between Frain's and Delaume's "author's image" and the "editorial ethos" of the two books. I show that their "argumentative dimension" manifests itself first of all at a paratextual/ peritextual level, and that the publishers exploit it to influence the reception of these works. Using the theoretical approaches of Literary Discourse Analysis and of Argumentation in Discourse, I also take into consideration the "ethos of the author" projected by Frain and Delaume in their books. The objectivity/subjectivity of their discourse, as well as the rhetoric strategies they use, are analysed in order to revisit the complex and gradual character of the notion of "argumentative dimension", in relation to the plurisemiotic potential of the digital text.

\section{INDEX}

Mots-clés : discours plurisémiotique, ethos auctorial, ethos éditorial, image d'auteur, littérature numérique

Keywords : digital literature, ethos of the author, image of the author, plurisemiotic discourse, publishing ethos 
AUTEUR

SARA AMADORI

Université de Bologne 